\title{
Acquisition Performance of Gold Sequence Based DS-CDMA Systems
}

\author{
Yong-Hwan Lee and Seung-Jun Kim \\ School of Electrical Engineering, Seoul National University, \\ San 56-1 Shinlim-Dong Kwanak-Ku, Seoul 151-742, Korea \\ TEL:+82-2-880-8413 FAX:+82-2-888-8213
}

\begin{abstract}
The performance of sequence acquisition for Gold sequence based DS-CDMA systems is studied in this paper. We consider the use of a sliding correlator-type structure for the acquisition scheme. Since acquisition of a PN sequence in DS-CDMA systems can be formulated as testing two simple hypotheses, we apply fixed sample size (FSS) for the synchronization test. The acquisition schemes require the knowledge of the partial correlation of Gold sequences, which is difficult to model. We propose the use of an approximate upper bound and then further linearize it for ease of designing the test scheme. The acquisition performance of Gold sequence based DS-CDMA systems is analyzed and is compared to that of $m$-sequence based ones. Numerical results indicate that the use of Gold sequences is very suitable for DS-CDMA systems. In addition, analytical results are verified by computer simulation.
\end{abstract}

\section{INTRODUCTION}

There has been extensive study on acquisition of spreading sequences for spread spectrum systems (e.g., see references $[1,2])$. Many techniques have been proposed, but most of them have been done in a single user environment, where maximal length sequences ( $m$-sequences) are usually used as the spreading pseudonoise (PN) sequence. For an efficient DS-CDMA system, it is necessary for the spreading $P N$ sequence to have good crosscorrelation characteristics as well as good autocorrelation characteristics. Although $m$-sequences have nice autocorrelation properties, the number of $m$ sequences that have good crosscorrelation properties is very small [3]. Hence, $m$-sequences may not be suitable for CDMA applications.

Orthogonal codes like Walsh codes have been employed for commercial CDMA systems [4]. However, the use of such codes is mostly limited to short length sequence spreading due to decoding complexity. Moreover, since the code is completely known, it may have some vulnerability in security aspects. Gold and Kasami sequences may provide large number of good sequences useful for CDMA applications. An acquisition scheme was studied for Kasami sequence based CDMA systems by taking advantage of the algebraic properties of Kasami sequences [5]. Gold sequences are applied to the Code Division Testbed (CODIT) system which uses short length Gold code as the spreading sequence. As the bandwidth of a CDMA system increases, it is required to use a PN spreading sequence having a longer period. But not much study has been done on acquisition of Gold sequence used in CDMA systems.

In this paper, we analyze acquisition performance for a DS-CDMA system employing Gold code for the spreading sequence. We assume that the data spread by a Gold sequence is transmitted by BPSK modulation over an additive white Gaussian noise (AWGN) channel, i.e., there is no jamming, fading, or other intentional interference signals, and that the received signal is coherently demodulated. A sliding correlator-type [6] is used for the acquisition scheme. The output of the correlator is tested by fixed sample size (FSS) test for detecting synchronization of two PN sequences.

\section{DS-CDMA SYSTEM EMPLOYING GOLD SEQUENCE}

In the receiver, the signal trasmitted by the $k$-th user in a DS-CDMA system with BPSK modulation can be expressed by

$$
s_{k}(t)=\sqrt{2 P_{k}} d_{k}\left(t-\tau_{k}\right) c_{k}\left(t-\tau_{k}\right) \cos \left(\omega_{c} t+\phi_{k}\right)
$$

where $\tau_{k}, \phi_{k}, \omega_{c}$ and $P_{k}$ denote the propagation delay, the phase of the carrier, the angular frequency of the carrier, and the signal power of the $k$-th user, respectively. $d_{k}(t)$ is the BPSK modulated $k$-th user data signal and $c_{k}(t)$ is the spreading signal of the $k$-th user represented by

$$
c_{k}(t)=\sum_{i=-\infty}^{\infty} c_{i}^{(k)} P_{T_{c}}\left(t-i T_{c}\right)
$$

where $c_{i}^{(k)}$ denotes the $i$-th chip value of Gold spreading sequence having a value of 1 or -1 , and $P_{T_{c}}(t)$ is a rect- 


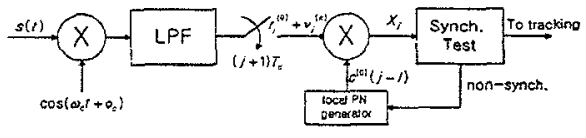

Figure 1. Block diagram of coherent acquisition scheme

angular pulse of amplitude 1 and duration of the chip time $T_{c}$ seconds.

Assume that there are $(K+1)$ users in the system and the signal of the zeroth user, $s_{0}(t)$, is the one to be demodulated as the target user signal. The signal received over an AWGN channel can be expressed by

$$
s(t)=s_{0}(t)+\sum_{k=1}^{K} s_{k}(t)+n(t)
$$

where the second term on the right hand side in (3) is the signal terms from $K$ other users, called the multiple user interference (MUI) or multiple access interference (MAI), and $n(t)$ is the channel noise. Since the MAI is normally much stronger than the channel noise even when the SNR is not high, we can neglect the AWGN term. Further assume that no data are modulated during the acquisition period, say $d_{0}(t)=1$, and the symbol time $T$ is an integer multiple of $T_{c}$.

Since the propagation delay can be written as

$$
\tau_{k}=l_{k} T_{c}+\gamma_{k} T_{c},
$$

where $l_{k}$ is an interger and $0 \leq \gamma_{k}<1, k=0,1, \cdots, K$, the signal terms can be expressed as

$$
\begin{aligned}
s_{0}(t)= & \sqrt{2 P_{0}} \sum_{i=-\infty}^{\infty} c_{i-l_{0}}^{(0)} P_{T_{c}}\left(t-\left(i+\gamma_{0}\right) T_{c}\right) \cos \left(\omega_{c} t+\phi_{0}\right) \\
s_{k}(t)= & \sqrt{2 P_{k}} \sum_{i=-\infty}^{\infty} d_{i-l_{k}}^{(k)} c_{i-l_{k}}^{(k)} P_{T_{c}}\left(t-\left(i+\gamma_{k}\right) T_{c}\right) \\
& \cdot \cos \left(\omega_{c} t+\phi_{k}\right), \quad k \neq 0 .
\end{aligned}
$$

The received signal is coherently demodulated as shown in Fig. 1. For ease of analysis, assume that the power of received signal is perfectly controlled, say $P_{k}=1, \forall k$ and $\phi_{k}=\phi_{0}, \forall k \neq 0$. Note that the latter condition results in the MAI to have the most conservative value. Further assume that the acquisition system knows the chip timing and let $\gamma_{0}=0$. Under these assumptions, the demodulated signal sampled at $t=(j+1) T_{c}$ is

$$
\begin{aligned}
r_{j} & =\frac{\sqrt{2}}{T_{c}} \int_{j T_{c}}^{(j+1) T_{c}} s(t) \cos \left(\omega_{c} t+\phi_{0}\right) d t \\
& =c_{j-l_{0}}^{(0)}+\sum_{k=1}^{K}\left[\left(1-\gamma_{k}\right) d_{j-l_{k}}^{(k)} c_{j-l_{k}}^{(k)}+\gamma_{k} d_{j-l_{k}-1}^{(k)} c_{j-l_{k}-1}^{(k)}\right] \\
& =r_{j}^{(0)}+\nu_{j}^{(K)},
\end{aligned}
$$

where the signal term $r_{j}^{(0)}$ and the MAI term $\nu_{j}^{(K)}$ are

$$
\begin{aligned}
r_{j}^{(0)} & =c_{j-l_{0}}^{(0)} \\
\nu_{j}^{(K)} & =\sum_{k=1}^{K}\left[\left(1-\gamma_{k}\right) d_{j-l_{k}}^{(k)} c_{j-l_{k}}^{(k)}+\gamma_{k} d_{j-l_{k}-1}^{(k)} c_{j-l_{k}-1}^{(k)}\right]
\end{aligned}
$$

respectively. The received sample after being multiplied by the local PN sequence is

$$
\begin{aligned}
x_{j} & =\left(r_{j}^{(0)}+\nu_{j}^{(K)}\right) c_{j-l}^{(0)} \\
& \triangleq \theta_{j}+\zeta_{j},
\end{aligned}
$$

where $l$ is the phase of the local PN generator, and $\theta_{j}$ and $\zeta_{j}$ are the target signal and the MAI terms correlated with the local PN signal over a chip time interval, respectively, and are given by

$$
\begin{aligned}
\theta_{j} & =r_{j}^{(0)} c_{j-l}^{(0)}=c_{j-l_{0}}^{(0)} c_{j-l}^{(0)} \\
\zeta_{j} & =\sum_{k=1}^{K}\left[\left(1-\gamma_{k}\right) d_{j-l_{k}}^{(k)} c_{j-l_{k}}^{(k)}+\gamma_{k} d_{j-l_{k}-1}^{(k)} c_{j-l_{k}-1}^{(k)}\right] c_{j-l}^{(0)}
\end{aligned}
$$

We consider the use of a sliding correlator structure for the acquisition scheme. Since the chip timing is assumed to be known, it suffices to update the phase of the local PN generator by $T_{c}$. To test synchronization of the two spreading sequences, let the null hypothesis $H_{0}$ be the case when the synchronization does not happen, i.e., $l \neq l_{0}$, and the alternative hypothesis $H_{1}$ be the case when $l=l_{0}$.

If $K$ is not too small, $\zeta_{j}$ can be approximated as a Gaussian random variable by the Central Limit Theorem. Assuming that $E\left\{d^{(k)}\right\}=0$ for all $k$ and $\left\{\gamma_{k}\right\}$ is uniformly distributed over interval $[0,1)$, it can be easily shown that $E\left\{\zeta_{j}\right\}=0$ and $\operatorname{var}\left\{\zeta_{j}\right\}=\frac{2}{3} K$. That is, the MAI signal can be approximated as a zero-mean Gaussian random variable with variance $\frac{2}{3} K$. The samples $\left\{x_{j}\right\}, j=1,2, \cdots$, are independent but not identically distributed under the hypothesis $H_{0}$ because $\theta_{j}$ depends on $j$. For the design and analysis of acquisition performance, we need to derive a simple model of the partial correlation of Gold sequences, which will be described in the next section.

\section{PARTIAL CORRELATION OF GOLD SEQUENCES}

Given a preferred pair of $m$-sequences $\left\{a_{i}\right\}$ and $\left\{b_{i}\right\}$ of period $N=2^{m}-1$ for an integer $m$, a. set of Gold sequences can be generated by

$$
\begin{aligned}
& g_{i}^{(0)}=a_{i}, \quad g_{i}^{(1)}=b_{i}, \\
& g_{i}^{(k)}=a_{i} \oplus b_{i-k}, \quad k=2,3, \cdots, N+1,
\end{aligned}
$$

thus yielding $(N+2)$ sequences. Here $\oplus$ denotes modulo2 addition. A set of spreading sequences, $\left\{c_{i}^{(k)}\right\}$, can be constructed by choosing $(K+1)$ sequences from $\left\{g_{i}^{(k)}\right\}$. 
Let $R_{w}^{(k)}(i, l)$ be the partial correlation function of a Gold sequence defined by

$R_{w}^{(k)}(i, l)=\sum_{j=1}^{w} c_{j-i}^{(k)} c_{j-l}^{(k)}, \quad 0 \leq k \leq K, 0 \leq i, l \leq N-1$.

From the properties of Gold sequences [3], when $i \neq l$, the correlation function over the full period of a sequence has one of the three values; for all $0 \leq k \leq K$,

$$
R_{N}^{(k)}(i, l)=\left\{\begin{array}{l}
-1 \\
t(m)-2 \equiv R_{\max } \\
-t(m) \equiv R_{\min }
\end{array}\right.
$$

where $t(m)=1+2^{\lfloor(m+2) / 2\rfloor}$. Here $\lfloor x\rfloor$ denotes the largest integer that does not exceed $x$. Gold sequences can be easily shown to have the following property

$$
\left\{c_{i-l_{k}}^{(k)} c_{i-l}^{(k)}\right\}=\left\{c_{i-l_{n}}^{(n)}\right\}
$$

for some $n$ and $l_{n}$, when $l_{k} \neq l$. Here $n$ and $l_{n}$ are determined by $k, l_{k}$ and $l$. For these $n$ and $l_{n}$, we can write

$$
R_{w}^{(k)}\left(l_{k}, l\right)= \begin{cases}\sum_{i=1}^{w} 1=w, & \text { if } l_{k}=l \\ \sum_{i=1}^{w} c_{i-l_{n}}^{(n)}, 0 \leq n \leq N+1, & \text { if } l_{k} \neq(17)\end{cases}
$$

When $l_{k}=l$ (under the hypothesis $H_{1}$ ), the partial correlation value increases in proportion to the correlation size $w$. When $l_{k} \neq l$ (under the hypothesis $H_{0}$ ), however, it is not easy to explicitly represent the value of $R_{w}^{(k)}\left(l_{k}, l\right)$. Therefore we need to characterize the partial correlation in a simple form.

We consider the three cases of $R_{N}=R_{\max }, R_{N}=-1$ and $R_{N}=R_{\min }$ separately. When $R_{N}=R_{\max }$, the mean and the variance of the partial correlation can be expressed as

$$
\begin{aligned}
E\left\{R_{w}^{(k)}\left(l_{k}, l\right)\right\} & =\sum_{i=1}^{w} E\left\{c_{i-l_{n}}^{(n)}\right\}=\frac{R_{\max }}{N} \cdot w \\
\operatorname{var}\left\{R_{w}^{(k)}\left(l_{k}, l\right)\right\} & =\sum_{i=1}^{w} \sum_{j=1}^{w} E\left\{c_{i-l_{n}}^{(n)} c_{j-l_{n}}^{(n)}\right\}-\frac{R_{\max }^{2}}{N^{2}} w^{2} \\
& =w\left[1+(w-1) \varepsilon-\frac{R_{\max }^{2}}{N^{2}} w\right],
\end{aligned}
$$

where $\varepsilon=E\left\{c_{i-l_{n}}^{(n)} c_{j-l_{n}}^{(n)}\right\}$, for $i \neq j$. Noting that when $w=N$, the variance should be zero, the variance can be approximated by

$$
\operatorname{var}\left\{R_{w}^{(k)}\left(l_{k}, l\right)\right\} \approx w\left(1-\frac{w}{N}\right),
$$

for a large $N$. For a Gold sequece with large period $N$, each chip of the sequence can be regarded as an i.i.d. random variable. Therefore, by invoking the Central Limit Theorem, $R_{N}^{(k)}\left(l_{k}, l\right)$ can be approximated as a Gaussian random variable with mean $\frac{R_{\max }}{N} w$ and variance $w\left(1-\frac{w}{N}\right)$ when $R_{N}^{(k)}\left(l_{k}, l\right)=R_{\max }$.

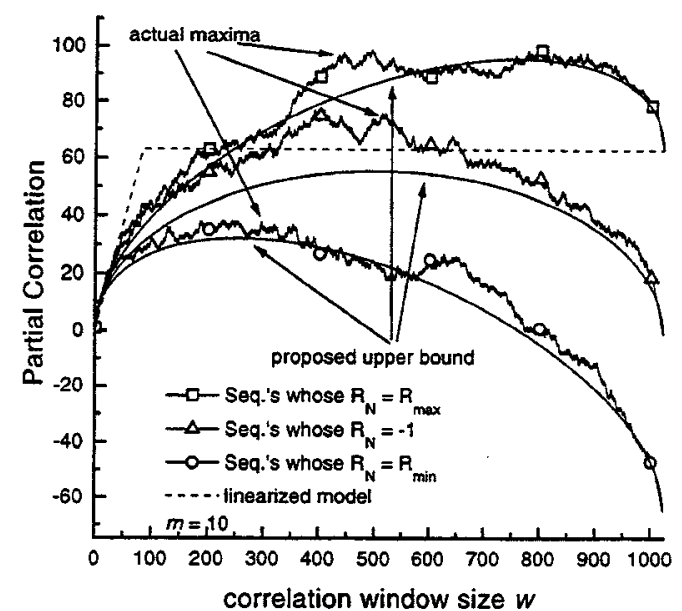

Figure 2. Partial correlation of Gold sequences under $H_{0}$ when $m=10$.

With the Gaussian approximation, we propose an approximate upper bound on $R_{w}$ under $H_{0}$ based on $99.98 \%$ confidence interval as

$$
\hat{R}_{w, R_{\max }}=\frac{R_{\max }}{N} w+3.5 \sqrt{w\left(1-\frac{w}{N}\right)},
$$

which is the mean plus three and half times of the standard deviation. Similarly, when $R_{N}^{(k)}\left(l_{k}, l\right)$ is equal to -1 and $R_{\min }$, we can formulate the corresponding upper bounds on $R_{w}$ by

$$
\begin{array}{r}
\hat{R}_{w,-1}=-\frac{w}{N}+3.5 \sqrt{w\left(1-\frac{w}{N}\right)} \\
\hat{R}_{w, R_{\min }}=\frac{R_{\min }}{N} w+3.5 \sqrt{w\left(1-\frac{w}{N}\right)} .
\end{array}
$$

Taking the worst case among (21), (22) and (23), we have an upper bound $\hat{R}_{w}$ for the partial correlation of the Gold sequences as

$$
\hat{R}_{w}=\hat{R}_{w, R_{\max }} .
$$

For conservative design, we will use this bound as the model of $R_{w}^{(k)}\left(l_{k}, l\right)$ under the hypothesis $H_{0}$.

The maximum partial correlation values of Gold sequences for all possible combination of $k, l_{k}$ and $l$, given correlation size $w$, are plotted in Fig. 2 for comparison to the upper bound (24). The tested Gold sequences are made up of from the preferred pairs of $m$-sequences generated by the generating polynomial in Table I. In this table, for example, $\{4,9\}$ represents a generating polynomial $1+z^{4}+z^{9}$ : The sequence is generated by adding the input bit to the outputs of the fourth and the ninth shift registers in a modulo- 2 mode. In this figure we

Table I. Generating polynomials used for Gold sequence generation

\begin{tabular}{|c|c|}
\hline$m$ & preferred pair \\
\hline 9 & $\{4,9\},\{1,2,4,5,6,9\}$ \\
10 & $\{3,10\},\{1,2,3,5,6,10\}$ \\
\hline
\end{tabular}


can see that the real maxima can exceed the proposed bound (24), but the rate by which this happens is on the order of $10^{-4}$ and has little effect on performance of the acquisition system. However, the curved shape of the proposed bound (24) implies that the corresponding samples under the hypothesis $H_{0}$ are no longer indentically distributed.

Since design and analysis become much more complicated when the samples are not i.i.d., we further approximate this into a two-piece linear model. The slope of the first piece is set to a value of 0.75 to accommodate the case when the phase of the local PN sequence is updated by $0.5 T_{c}$. The second piece has zero slope, i.e., it is set to a constant equal to $R_{\max }$. Denoting the crossing point of the two lines by $W_{b}$, the proposed piecewise linear model is given by

$$
\bar{R}_{w}= \begin{cases}0.75 w, & w \leq W_{b} \\ R_{\max }, & w>W_{b},\end{cases}
$$

where $W_{b}$, determined by $W_{b}=\frac{4}{3} R_{\max }$, is rounded up to the nearest integer. This approximate model, depicted in Fig. 2 as a dotted line, is used for modeling the hypothesis $H_{0}$. From Eq. (11), it can be seen that $\sum_{j=1}^{w} \theta_{j}=R_{w}^{(0)}\left(l_{0}, l\right)$. Therefore the signal model $\hat{\theta}$ for $\theta_{j}$ can be set up under hypotheses $H_{1}$ and $H_{0}$ by

$$
\begin{aligned}
& \hat{\theta}=1 \equiv \hat{\theta}_{1} \\
& \hat{\theta}=\hat{\theta}_{0 j}=\left\{\begin{array}{ll}
\hat{\theta}_{a}=0.75, & j \leq l_{0}=l\left(H_{0}\right) \\
\hat{\theta}_{b}=0.0, & j>W_{b}
\end{array} \quad ; l_{0} \neq l\left(H_{1}\right)\right.
\end{aligned}
$$

respectively.

\section{DESIGN OF COHERENT ACQUISITION SCHEME}

Using the signal model developed in the previous section, we can formulate the acquisition problem using Gold sequences as testing the two simple hypotheses:

$$
\begin{aligned}
& H_{0}: X_{j} \sim f_{n}\left(x-\hat{\theta}_{0 j}\right) ; \quad \hat{\theta}_{0 j}= \begin{cases}\hat{\theta}_{a}, & j \leq W_{b} \\
\hat{\theta}_{b}, & j>W_{b}\end{cases} \\
& H_{1}: X_{j} \sim f_{n}\left(x-\hat{\theta}_{1}\right),
\end{aligned}
$$

where $f_{n}(x)$ is a zero-mean Gaussian probability density function $(p d f)$ with variance $\sigma_{n}^{2}$. When there exist $K$ interfering users, $\sigma_{n}^{2}=\frac{2}{3} K$. From the log-likelihood ratio formed by

$$
\begin{aligned}
z_{j} & =\ln \frac{f_{n}\left(x_{j}-\hat{\theta}_{1}\right)}{f_{n}\left(x_{j}-\hat{\theta}_{0 j}\right)} \\
& = \begin{cases}\frac{\hat{\theta}_{1}-\hat{\theta}_{a}}{\sigma_{n}^{2}}\left(x_{j}-\frac{\hat{\theta}_{a}+\hat{\theta}_{1}}{2}\right), & j \leq W_{b} \\
\frac{\hat{\theta}_{1}-\hat{\theta}_{b}}{\sigma_{n}^{2}}\left(x_{j}-\frac{\hat{\theta}_{b}+\hat{\theta}_{1}}{2}\right), & j>W_{b}\end{cases}
\end{aligned}
$$

the FSS test is performed by:

$$
\lambda\left(W_{F}\right) \triangleq \sum_{j=1}^{W_{F}} z_{j}\left\{\begin{array}{l}
\geq t_{F} \Rightarrow \text { Accept } H_{1} \\
<t_{F} \Rightarrow \text { Accept } H_{0}
\end{array}\right.
$$

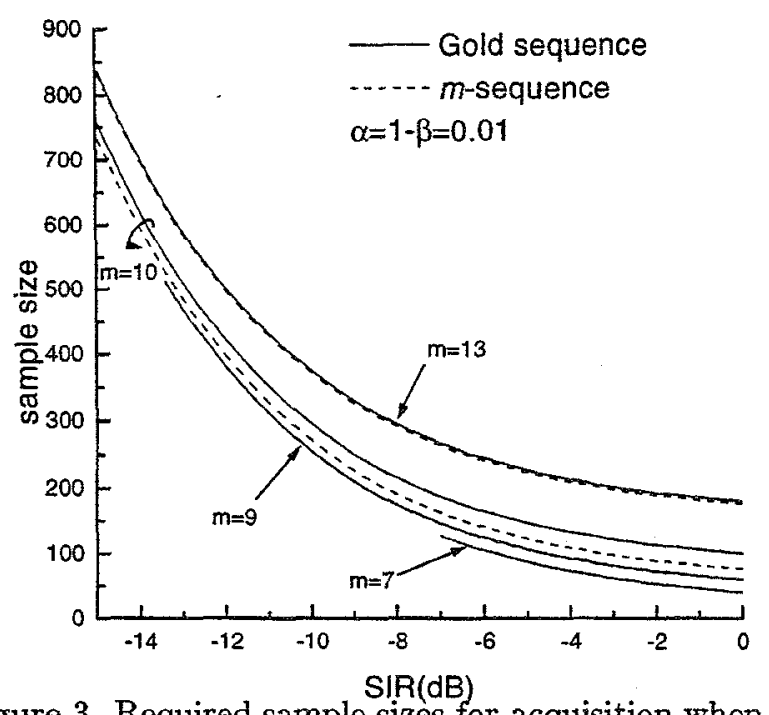

Figure 3. Required sample sizes for acquisition when $m=9, \alpha=0.01$

where the sample size $W_{F}$ and the threshold $t_{F}$ are determined so as to satisfy the prescribed false alarm probability $\alpha$ and the miss detection probability $(1-\beta)$. For convenience, define

$$
\begin{gathered}
E\left\{Z_{j} \mid \hat{\theta}=\hat{\theta}_{1}\right\} \equiv \begin{cases}\mu_{a}=\frac{1}{2}\left(\hat{\theta}_{1}-\hat{\theta}_{a}\right)^{2} / \sigma_{n}^{2}, & j \leq W_{b} \\
\mu_{b}=\frac{1}{2}\left(\hat{\theta}_{1}-\hat{\theta}_{b}\right)^{2} / \sigma_{n}^{2}, & j>W_{b}\end{cases} \\
\operatorname{var}\left\{Z_{j} \mid \hat{\theta}=\hat{\theta}_{1}\right\} \equiv\left\{\begin{array}{ll}
\sigma_{a}^{2}=\left(\hat{\theta}_{1}-\hat{\theta}_{a}\right)^{2} / \sigma_{n}^{2}, & j \leq W_{b} \\
\sigma_{b}^{2}=\left(\hat{\theta}_{1}-\hat{\theta}_{b}\right)^{2} / \sigma_{n}^{2}, & j>W_{b}
\end{array} .\right.
\end{gathered}
$$

The required sample size and the threshold are calculated by

$$
\begin{aligned}
W_{F} & =\frac{\sigma_{a}^{2}}{\left(2 \mu_{a}\right)^{2}}\left[\Phi^{-1}(\alpha)+\Phi^{-1}(1-\beta)\right]^{2} \\
t_{F} & =\frac{\sqrt{W_{F}} \sigma_{a}}{2}\left[\Phi^{-1}(1-\beta)-\Phi^{-1}(\alpha)\right]
\end{aligned}
$$

for $W_{F}<W_{b}$ and

$$
\begin{aligned}
W_{F}= & \frac{1}{8 \mu_{b}^{2}}\left[-8 \mu_{b} \mu_{w}+\sigma_{b}^{2} \rho^{2}\right. \\
& +\sqrt{\left.\left(8 \mu_{b} \mu_{w}-\sigma_{b}^{2} \rho^{2}\right)^{2}-16 \mu_{b}^{2}\left(4 \mu_{w}^{2}-\rho^{2} \sigma_{w}^{2}\right)\right]} \\
t_{F}= & \frac{1}{2} \sqrt{W_{F} \sigma_{b}^{2}+\sigma_{w}^{2}} \cdot\left[\Phi^{-1}(1-\beta)-\Phi^{-1}(\alpha)\right](33)
\end{aligned}
$$

for $W_{F} \geq W_{b}$, where $\Phi^{-1}(\cdot)$ is the inverse of the standard normal distribution function, $\mu_{w}=\left(\mu_{a}-\mu_{b}\right) W_{b}, \sigma_{w}^{2}=$ $\left(\sigma_{a}^{2}-\sigma_{b}^{2}\right) W_{b}$, and $\rho^{2}=\left[\Phi^{-1}(\alpha)+\Phi^{-1}(1-\beta)\right]^{2}$.

\section{PERFORMANCE EVALUATION}

When a sliding correlator scheme is used, it has been shown that the acquisition time is linearly proportional to the sample size of the test $[6,7]$. Therefore we evaluate acquisition performance in terms of the sample size required to satisfy given error probabilities $\alpha$ and $1-\beta$. Note that the sample sizes for the FSS test are generally not the same under $H_{0}$ and $H_{1}$, except when $\alpha=1-\beta$. For ease of comparison, we assume $\alpha=1-\beta$. 
To see feasibility of the use of Gold sequences for CDMA applications, acquisition performance of Gold sequence based DS-CDMA is compared to that of $m$ sequence based one. The required sample sizes for Gold sequence and $m$-sequence based systems are shown in Fig. 3. The sample size for $m$-sequence based system is calculated using the method proposed in [7]. It can be seen that little difference exists in the acquisition time between the two systems and suggests that Gold sequence is fairly suited for CDMA applications.

To verify validity of the design using the approximated model, analytical results are compared with simulation results. When the FSS test is applied for the detection scheme, detection probabilities at different SIRs are plotted in Fig. 4 as a function of sample size. In this figure, analytical performance is shown by lines and actual performance is shown by symbols. Under the hypothesis $H_{1}$, Fig. 4(a) shows that the analysis agrees very well with the simulation. Under the hypothesis $H_{0}$, Fig. 4(b) shows that the actual false detection probability is a little less than the analytical one but it always guarantees the prescribed ones. The small difference under $H_{0}$ is mainly be due to the use of a conservatively approximated model for the out-of-sync condition.

\section{CONCLUSION}

When Gold codes are used for the spreading sequence in a DS-CDMA system, acquisition performance has been analyzed. Since the partial correlation of Gold sequences is difficult to explicitly describe, an approximate upper bound has been proposed. For ease of design and analysis, the proposed bound has been further approximated as a piecewise linear model. Based on the approximate model, the FSS test is analytically designed for testing the alignment of two spreading sequences. Numerical results show that the use of Gold sequences is quite feasible for CDMA applications, since it does not degrade acquisition performance in comparison to the use of $m$ sequences. Finally, analytical results have been verified by computer simulation.

\section{REFERENCES}

[1] J. Holmes, Coherent Spread Spectrum Systems. New York: Wiley, 1982.

[2] M. Simon, J. Omura, R. Scholtz, and K. Levitt, Spread Spectrum Communications, vols. I, II, and III. Rockville, MD: Computer Science Press, 1985.

[3] D. Sarwate and M. Pursley, "Crosscorrelation Properties of Pseudorandom and Related Sequences," Proc. IEEE, vol.68, no.5, pp.593-619, May 1980.

[4] TIA/EIA Interim Standard-95, "Mobile StationBiase Station Compatibility Standard for DualMode Wideband Spread Spectrum Cellular System," July 1993.
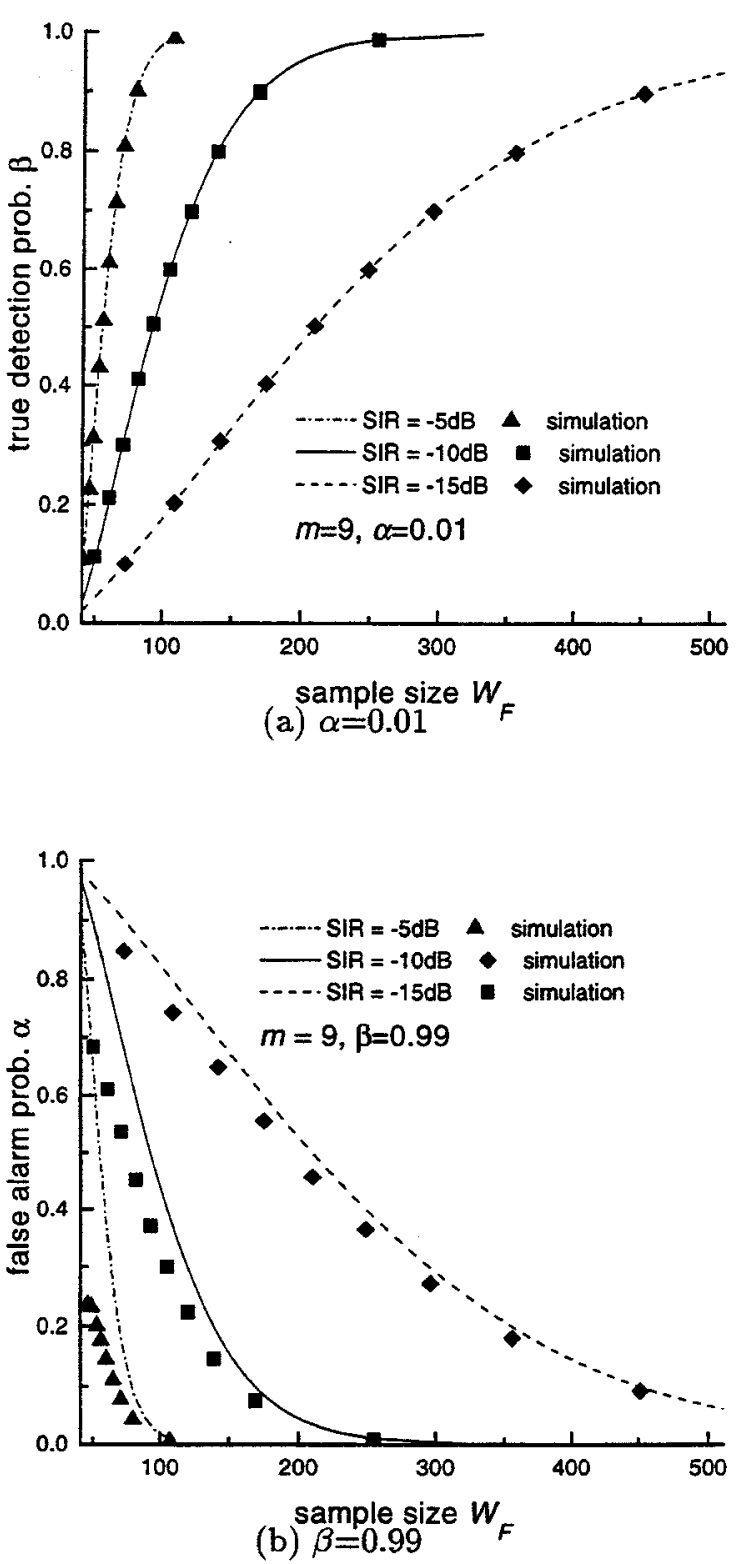

Figure 4. Acquisition performance using the FSS test when $m=9$.

[5] R. Barghouthi and G. Stüber, "Rapid Sequence Acquisition for DS/CDMA Systems Employing Kasami Sequences," IEEE Trans. on Commun., vol.42, no.2/3/4, pp.1957-1968, Feb./Mar./Apr. 1994.

[6] R. Pickholtz, D. Schilling and L. Milstein, "Theory of Spread-Spectrum Communications-A Tutorial," IEEE Trans. on Commun., vol.COM-30, no.5, pp.855-884, May 1982.

[7] Y.-H. Lee and S. Tantaratana, "Sequential Acquisition of PN Sequences for DS/SS Communications: Design and Performance," IEEE J. on Selected Areas in Commun., vol.10, no.4, pp.750-759, May 1992. 